\title{
Development and Prospect of Automobile Four-Wheel Steering System
}

\section{Yajie Chen*, Kehui Ma, Yongguo Zhang, Zhen Xu and Pengcheng Zhou}

\author{
School of Transportation and Vehicle Engineering, \\ Shandong University of Technology, Zibo, Shandong,255000, China \\ *Corresponding author details: Yajie Chen; chenyajie977@163.com
}

\begin{abstract}
Descriptions of the car four-wheel steering system development process, characteristics and advantages of four-wheel steering technology analysis of the current level of development and the control strategy, combined with electronically controlled four-wheel steering application of technology to the development direction of the steering system are discussed.
\end{abstract}

Keywords: Car wheel steering; Electrically control ledsteering technology; Development direction

\section{INTRODUCTION}

At present, the number of motor vehicles in China is growing faster and faster, while the expansion of urban roads lags behind obviously, and it is difficult for vehicles to turn and turn around on narrow roads, which brings great inconvenience to people. At the same time, China's expressways are developing rapidly. With the vigorous development of transportation, the number of expressway accidents is increasing year by year, especially on wet roads, where the vehicles are out of control due to the reduction of ground adhesion coefficient. While analyzing the events, people found that four-wheel steering technology can solve these two problems better. The front and rear four wheels of a four-wheel steering vehicle have steering function [3], and according to the different swing angles and phases of the front and rear wheels, the turning radius of the vehicle can be reduced or increased, thereby improving the flexibility or stability of the vehicle [4]. Therefore, the fourwheel steering car has two advantages compared with the traditional front-wheel steering car: at low speed, the deflection direction of the rear wheel is opposite to that of the front wheel, which reduces the turning radius and can realize more flexible steering; At high speed, the rear wheel deflects in the same direction as the front wheel, which increases the turning radius and improves the stability of the vehicle. Since 1980s, four-wheel steering technology has been applied to automobiles, but it has not been popularized. The reason is that the structure of four-wheel steering system is complex, and the requirements for electronic control technology are high, thus increasing the cost and reducing the reliability of steering system [6].

\section{DEVELOPMENT OF FOUR-WHEEL STEERING SYSTEM}

Four-wheel steering technology originated in Japan. In 1907, the Japanese government issued the first patent certificate on four-wheel steering. In 1962, Japan Automobile Engineering Association put forward the fourwheel steering technology of active rear wheel steering, and started the research of automobile four-wheel steering In 1985, Nissan successfully applied the world's first fourwheel steering system on buses.
The earliest four-wheel steering system adopts mechanical mechanism. The front and rear wheel steering systems are connected by an intermediate steering shaft. When steering, the steering wheel angle first acts on the front wheel steering system.

At the same time, the intermediate steering shaft is used to transmit the steering wheel angle to the rear wheel steering gear to control the rear wheel to rotate [8]. Subsequently, the electro-hydraulic four-wheel steering mechanism appeared. Both the front and rear wheels used hydraulic power steering. When the front wheel turned, the middle steering shaft transmitted the signal to the rear wheel power steering mechanism. In the rear wheel power steering mechanism, according to the ratio of front wheel angle, vehicle speed and rear wheel steering angle, the controller controls the motor to drive the power cylinder to act, thus changing the rotation direction and angle of the rear wheel. At present, the advanced electric four-wheel steering mechanism omits the middle steering shaft and hydraulic pipeline, which makes the structure simpler. In this mechanism, the current information is collected by the control unit, and the steering motors of the front and rear wheel steering systems are driven to steer, and the steering motors can be separately equipped for each wheel to realize four-wheel independent control [9].

\section{CONTROL OF FOUR-WHEEL STEERING SYSTEM}

In the design of the steering mechanism of wheeled vehicles, the steering system is required to meet the Ackerman steering principle, that is, four wheels can rotate around the same center in an ideal state without sideslip. At this time, all four tires are in a pure rolling state without sliding wear [10], as shown in Figure 1. The two steering wheels should satisfy the relation: $\cot \beta-\cot \alpha=\frac{M}{L}$

In which:

$\alpha$-inner wheel rotation angle;

$\beta$-outer wheel angle;

$\mathrm{M}$ - the center distance between two kingpins in the axial direction;

L-wheelbase of vehicle; 
FIGURE 1: ACKERMAN STEERING PRINCIPLE

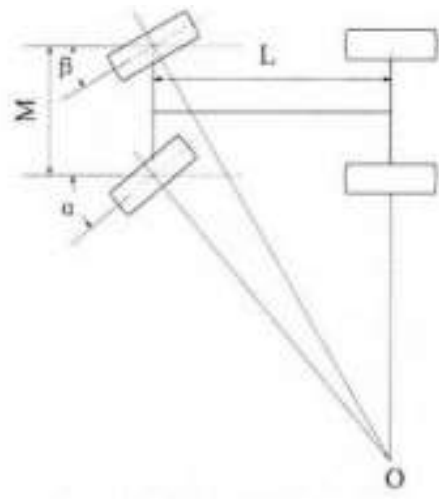

Traditional front-wheel steering vehicles use trapezoidal mechanism to satisfy Ackerman's principle. In the steering trapezium mechanism, the length of the bottom edge (the center distance between two kingpins) $m$ is given by the overall design of the vehicle, and the length of the two waists is equal. Therefore, there are only two independent variables to be determined, one is the length of the upper bottom edge (tie rod) and the other is the length of the two waists (trapezoidal arms), and these two parameters can also be converted into the trapezoidal bottom angle $\theta$ and waist length m, as shown in Figure 2 [11].

FIGURE 2: STEERING TRAPEZOIDAL MECHANISM

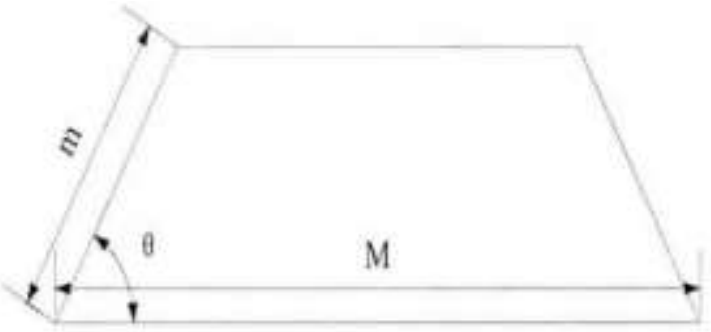

Generally, in design, according to the value of $\theta$ and $m$, a set of actual corresponding values of the selected mechanism parameters within the steering wheel angle range $(\alpha<$ $\alpha_{\max }$ ), and the inner wheel angle $\alpha$ and outer wheel angle $\beta$ are made by using the graph method, and the actual characteristic curve of this set of corresponding angles $\left(\alpha_{i}, \beta_{i}\right)$ is made as shown in Figure 3 , and compared with the theoretical characteristic curve, and the deviation value of the angle $\triangle \beta[12]$ is obtained. The straight-line $\mathrm{GF}$ is the theoretical characteristic curve determined by Ackermann principle, and the arc line GE is the actual characteristic curve determined by trapezoidal mechanism parameters. If the two characteristic curves are close, the maximum deviation value $\Delta \beta_{\max }$ [13]. It is less than the allowable deviation, indicating that the steering trapezoidal geometric parameters are selected reasonably; If $\Delta \beta_{\max }$ is greater than the allowable deviation, the trapezoidal parameter must be re-selected [3].

FIGURE 3: PARAMETER DETERMINATION OF TRAPEZOIDAL MECHANISM

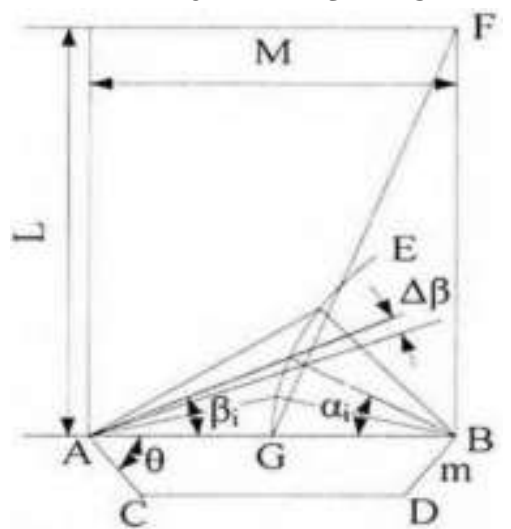

It can be seen that the trapezoidal steering mechanism can only approximately satisfy Ackerman's principle within a certain range, and reduce tire wear during turning. However, in the design of four-wheel steering vehicle, the difference of rear wheel swing angle between high and low speed steering causes the change of steering wheelbase, which makes trapezoidal steering mechanism unsuitable for four-wheel steering vehicle [14]. As shown in Figure 4 and Figure 5, a four-wheel steering vehicle should meet the following requirements:

$$
\left\{\begin{array}{l}
\cot \beta_{1}-\cot \alpha_{1}=\frac{M}{L_{1}} \\
\cot \beta_{2}-\cot \alpha_{2}=\frac{M}{L_{2}}
\end{array}\right.
$$

Because L1 and L2 both change when the four-wheel steering vehicle changes between high speed and low speed, and L changes greatly, it will bring great difficulties to the design of four-wheel steering vehicle [15].

FIGURE 4: STATES OF LOW-SPEED TURNING WHEELS

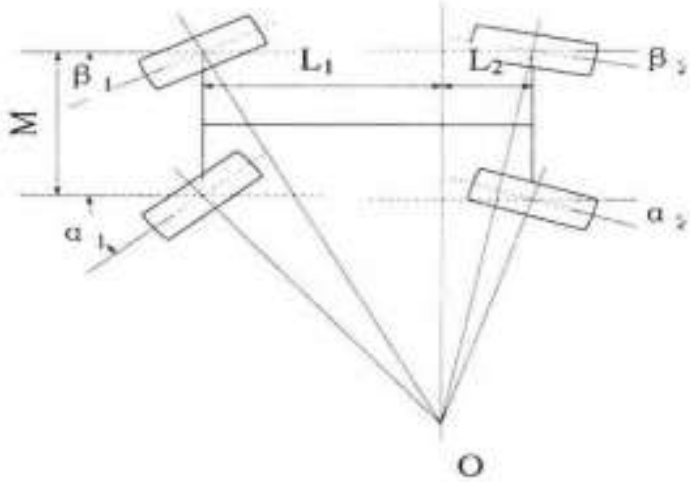

FIGURE 5: STATES OF EACH WHEEL IN
HIGH-SPEED TURNING

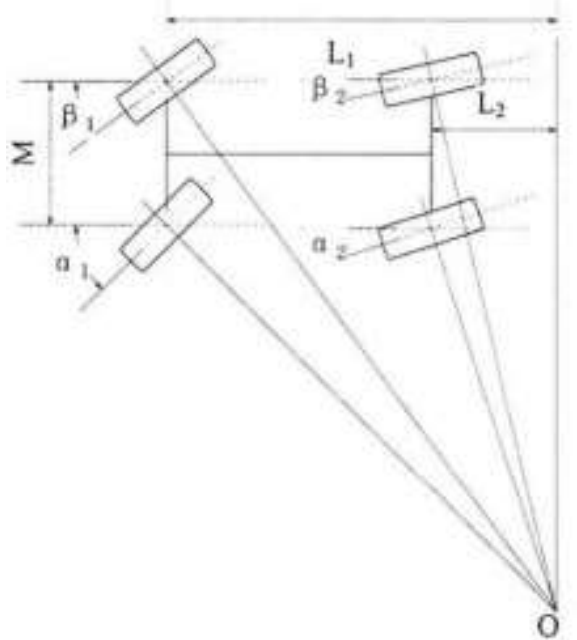

Some four-wheel steering systems add another steering device to the rear wheels of automobiles on the basis of front wheel steering, and they are connected in a certain way, so that the rear wheels also participate in steering while the front wheels turn. Although this four-wheel steering system has simple structure and high reliability, it cannot meet Ackerman principle [16], which leads to serious tire wear, increased driving resistance and increased fuel consumption. Because the turning angle of the rear wheel can realize in-phase steering and reverse steering at high speed and low speed, the turning radius of four-wheel steering vehicle changes greatly, and if the traditional trapezoidal steering mechanism is adopted, there will be a big error, so it is necessary to control each tire independently to satisfy Ackerman's principle [18]. 
In recent years, electronic technology has been widely used in automobile field, and with the improvement of reliability of electronic equipment, some complicated mechanical structures have been gradually replaced by electronic structures. For the automobile steering system, after years of research and practice, the electronic control steering technology has appeared at present [17]. In 2012, Nissan applied electronic steering technology to "British-finidi Q50" cars, becoming the first enterprise to use electronic steering technology on production cars. The electronic steering system cancels the mechanical connection between steering wheel and steering gear, and uses electric signals and motors to control the steering of tires. Firstly, the sensor is used to detect the turning angle of the steering wheel, and then the ECU (Electronic Control Unit) converts the angle that the tire should turn into a control signal, which is transmitted to the motor that controls the tire steering through the internal wiring harness of the automobile to realize steering. Because the steering wheel operation is transmitted through the wire harness instead of the steering shaft, it is called electronic steering [4]. At the same time, in order to ensure the reliability of the steering system, the mechanical connection between the steering wheel and the steering gear in the traditional steering system remains. It can be seen that the application of electronic control steering technology in traditional front-wheel steering vehicles seems to be "redundant", but it is of great significance for four-wheel steering vehicles. With the electronic control steering technology as the foundation, four-wheel independent steering can be realized quickly, and four-wheel independent steering is the key for four-wheel steering vehicles to meet Ackerman's principle.

\section{CONTROL STRATEGY OF FOUR-WHEEL STEERING SYSTEM}

At present, there are mainly two control methods of four-wheel steering system, which are divided into angle following type (Figure 6) and speed sensing type (Figure 7).

FIGURE 6: CORNER FOLLOWING TYPE

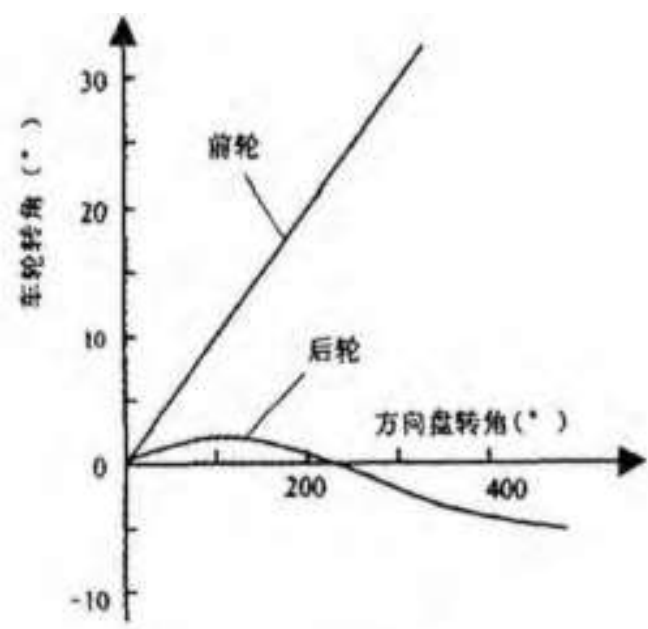

The characteristic of the angle following type is that the deflection direction and angle of the rear wheel are controlled by the angle of the steering wheel [19]. When the steering wheel angle is large, the rear wheel deflects oppositely relative to the front wheel, and it is considered that the vehicle speed is low at this time; When the steering wheel angle is small, the rear wheel deflects in the same direction as the front wheel, and it is considered to be in a high-speed driving state. However, this system does not take into account the possibility of human mis operation, which will deteriorate the handling stability of the automobile when turning sharply at high speed, so it is rarely used [20].
The characteristic of the speed sensing type is that the direction and angle of the rear wheel deflection are mainly controlled by the speed of the vehicle, and are also comprehensively controlled by dynamic parameters such as the front wheel angle, lateral acceleration and yaw rate. The front and rear wheels deflect in the reverse direction when the car turns at low speed, and deflect in the same direction when turning at high speed [31]. This system comprehensively considers the influence of various dynamic parameters of the automobile on the steering stability of the automobile, and is the main control method used in the four-wheel steering automobile at present.

\section{FIGURE 7: SPEED SENSING TYPE}

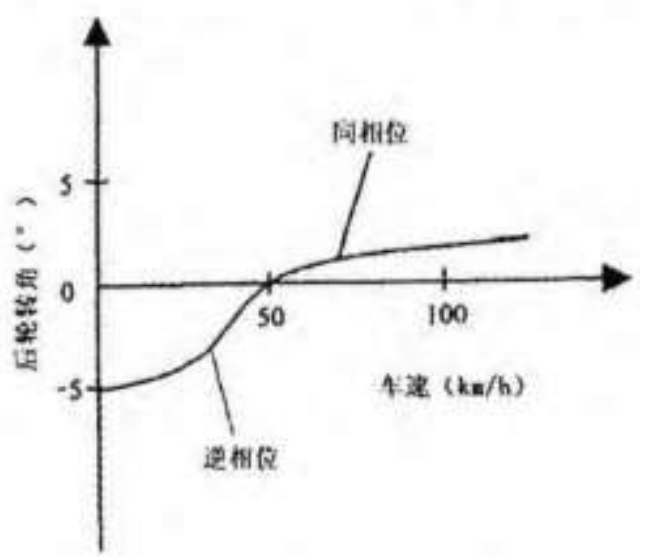

The main control objectives of four-wheel steering system are to reduce the phase difference between lateral acceleration and yaw rate and their respective phases; Reduce the sideslip angle at the center of mass of the car. At present, the control methods used in some four-wheel steering vehicles mainly include: four-wheel steering system with a certain steering ratio between front and rear wheels; The steering ratio of front and rear wheels is a function of the front wheel angle; The steering ratio of front and rear wheels is a function of vehicle speed. Four-wheel steering system with first-order lag: Four-wheel steering system with reverse phase characteristics; Four-wheel steering with optimal control characteristics: Self-adaptive four-wheel steering etc. [33]. Of course, besides the objective control method established by these dynamic parameters, the subjective driving feeling of drivers should also be considered. At present, the steering mode used by drivers is traditional front-wheel steering, whose turning radius has a linear proportional relationship with steering wheel angle, while four-wheel steering cars have smaller turning radius at low speed and larger turning radius at high speed, which is quite different from the driving experience of front-wheel steering cars. Therefore, for the research of four-wheel steering vehicle control strategy, it is necessary to consider the driver's driving experience as an important element. Starting from subjective evaluation, in the high-low speed transition stage, the driver's insensitive transition mode should be adopted as far as possible to keep the unity of subjective evaluation and objective evaluation [43].

\section{OUTLOOK}

APPLICATION OF FOUR-WHEEL STEERING COMBINED WITH HUB MOTOR

With many advantages, the hub motor is considered as the main driving form of electric vehicles in the future, and it has been a research hotspot at home and abroad. As the power source of electric vehicles, the hub motor itself has a series of advantages, including: fast response speed, high torque control accuracy, providing driving and braking torque, independent torque control, long service life, etc. 
The hub motor is directly installed in the driving wheel, so there is no need to design the transmission, universal transmission, differential and other traditional transmission components, which will bring great changes and optimization to the design and control of the electric vehicle chassis, including:

(1) The system efficiency is improved, and the hub motor drive system is more than $10 \%$ higher than the centralized motor drive efficiency;

(2) The torque response precision is high and the response speed is fast, which can realize the independent control of distributed driving wheels;

(3) The freedom of chassis layout is high, and the lightweight degree of the whole vehicle is greatly improved; It is the preferred power source for hybrid electric vehicles, pure electric vehicles and fuel cell vehicles;

(4) It is beneficial to realize more optimized distributed driving and braking control, and is more convenient to realize the upper control strategy of automatic driving [51].

The advantage of independent torque control of hub motor combined with four-wheel steering is beneficial to the realization of Ackerman principle.

\section{APPLICATION OF FOUR-WHEEL STEERING IN AUTOMATIC DRIVING}

With the application of electronic control steering technology, the development of four-wheel steering technology has taken a big step forward. In recent years, automobile active safety technology has developed rapidly, mainly including ABS anti-lock braking system, ASR drive anti-skid control system, EBD electronic braking force distribution system, TCS traction control system, ESP automobile dynamic control system, EBA emergency braking auxiliary system, ACC adaptive cruise control system, EMB electronic mechanical braking system, etc. Most of these active safety technologies are developed based on the control of the braking system. By detecting the running state of the vehicle, they intervene in the braking of each wheel when the vehicle is about to slip or roll over, so as to get the vehicle out of danger [57].

At present, automatic driving technology is a very popular research direction in the automotive field, and the future development of automobiles must be intelligent and networked. Automatic driving is applied to traditional steering system, and steering flexibility is limited to some extent. Can't give full play to the flexibility of turning. If automatic driving is combined with four-wheel steering, it will definitely increase the steering flexibility of automobiles, and can realize lateral parking or even 360 steering, which is more conducive to the intelligent realization of automatic driving.

\section{REFERENCES}

[1] Gao Qi, Wang Chunyan. Research on lateral stability control of four-wheel drive vehicle under steering condition. Journal of Chongqing University of Technology (Natural Science Edition), 2019,33(8):16-21,37.

[2] Cui Ning, Liu Xiangyang, Ma Lei. Linear optimization design of steering controller for four-wheel independent steering vehicle. Mechanical Science and Technology, 2019,38(2):304-310.
[3] Ran Tao, Deng Lindong. Simulation analysis of steering motion of four-wheel-drive electric vehicle. Auto time, 2019, (10):66-67.

[4] Zhou Su, Wu Nan, Zhi Xuelei. Path tracking predictive control of four-wheel independent steering electric vehicle. Journal of Tongji University (Natural Science Edition), 2019,47(6):842-850.

[5] Li Gang, Li Gaochao, Lei Yongqiang, et al. Research on integrated control of four-wheel steering and yaw moment of four-wheel independent steering/independent electric drive vehicle. Mechanical Design and Manufacturing, 2019, (5):147-150,154.

[6] Ma Shiran, Gong Guoqing, Chen Yong, et al. Steering stability control of four-wheel motor electric vehicle. Computer simulation, 2018,35(12):117-121.

[7] Hang Peng, Chen Xinbo, Zhang Bang, et al. Active obstacle avoidance path planning and tracking control for four-wheel independent steering and independent drive electric vehicles. Automotive engineering, 2019,41(2):170-176.

[8] Chen Zheming, Zhou Peng, Chen Bao, et al. Research on stability control strategy of automobile fourwheel independent steering. Computer simulation, 2018,35(7):93-97,147.

[9] Wang Yong. Research on the corner algorithm of fourwheel independent steering vehicle based on vector operation. Lifting and Transportation Machinery, 2018, (12):113-115.

[10] Yu Xia, Meng Xianjie. Establishment and simulation analysis of two-degree-of-freedom model of fourwheel steering system. Auto time, 2019, (12):7-8.

[11] Li Gang, Lei Yongqiang, Ma Gaofeng. Coordinated control of yaw moment and active steering of fourwheel drive electric vehicle. Mechanical Design and Manufacturing, 2018, (8):229-232.

[12] Kunshan weikai children's products co, ltd. A front wheel synchronous steering adjustment structure for four-wheeled baby carriage: CN201920187275.9.2019-10-22.

[13] Anne Girl, Lian Yangyang. Several problems in steering system design of front-wheel steering fourwheel drive tractor. Super Science, 2018, (6):220.

[14] Shandong University of Technology. In-wheel motor electric vehicle with four-wheel independent steering function: CN201910278205.9.2019-08-06.

[15] Dongfeng motor group co, ltd. Differential torsional yaw moment distribution method for improving steering performance of four-wheel hub motor drive: CN201910550327.9.2019-10-15

[16] Fang Chunjie. Active steering control and simulation of four-wheel steering vehicle. Automotive Industry Research, 2018, (3):56-59.

[17] Wang Lamei. Research on Steering Control Strategy of Four-wheel Independent Electric Drive Vehicle. Southern Agricultural Machinery, 2018,49(4):188.

[18] Shandong artu robot technology co, ltd. Four-wheel independent drive steering full-swing mobile chassis: CN201822128388. X.2019-08-20. 
[19] Wang Shuai, Zhang Jinyang, Sun Yifan, et al. Design of four-wheel drive in-situ steering chassis. Mechanical Engineering and Automation, 2017, (5):100-101,104.

[20] Song Xunxun, Song Mingxiang. Talking about the working principle and characteristics of four-wheel steering. Electronic Journal of New Education Times (Student Edition), 2018, (12):242.

[21] Liaoning University of Technology. A steering stability method based on four-wheel independent drive electric vehicle: CN201910596951.2.2019-10-25.

[22] Liaoning University of Technology. A steering transmission device for four-wheel independent drive and steering: CN201821950014. X.2019-08-20.

[23] Qiao Jianlu, Fan Ying, Jin Minjie, et al. Study on steering characteristics of four-wheel steering vehicles. Journal of north University of China (Natural Science Edition), 2017,38(4):458-465.

[24] Dalian university of technology. Torque distribution method of four-wheel independent drive electric vehicle under steering condition: CN201910602546.7.2019-10-01.

[25] Hu Yahui. Research on Differential Steering Performance of Four-wheel Independent Drive Electric Vehicle. Shaanxi: Xi 'an Polytechnic University, 2019.

[26] Suzhou Automotive Research Institute of Tsinghua University (Wujiang). A steering stability control method for four-wheel drive electric vehicles: CN201910566687.8.2019-09-20.

[27] Nanjing University of aeronautics and astronautics. A method of four-wheel motor distributed driving vehicle to turn in situ: CN201910389448. X.2019-0917.

[28] Nanjing University of aeronautics and astronautics. A four-wheel active steering electric wheel system by wire and its steering fault-tolerant control method: CN201910057136.9.2019-05-21.

[29] Li Gang, Ji Xiao, Xu Chunsheng, et al. Summary of Key Technologies of Personalized Intelligent Control for Distributed Drive Electric Vehicles. Journal of Liaoning University of Technology (Natural Science Edition), 2019,39(6):375-380

[30] Zheng Zhu'an, Ma Shicheng, Lv Hongming, et al. Frame design and analysis of electric formula racing car based on in-wheel motor. Agricultural Equipment and Vehicle Engineering, 2019,57(11):15-18,32.

[31] Tian Zhaoxian, Yang Kun, Wang Jie, et al. Research on electronic differential control of rear-wheel-motor electric vehicle. Agricultural Equipment and Vehicle Engineering, 2019,57(10):6-10.

[32] Liu Gang, Xu Wenbo, Jin Liqiang. Research on pressure estimation and control method of hydraulic actuator of electric vehicle driven by hub motor. Automotive Engineering, 2019,41(10):1138-1144.

[33] Zhao Qijin, Liao Zili, Zhang Xinxi, et al. Research on sensorless control of hub motor of electric drive vehicle. Fire and Command Control, 2019,44(8):4145,50 .
[34] Huang Caixia, Lei Fei, Hu Lin, et al. Lateral stability control of hub motor driven vehicle with regional pole assignment. Automotive Engineering, 2019,41(8):905-914.

[35] Mao Pengtao. Summary of key technologies of hub motors for new energy electric vehicles. Digital users, 2019,25(21):176.

[36] Wang Zhenpo, Ding Xiaolin, Zhang lei. Overview of key anti-skid control technologies for four-wheel hub motor-driven electric vehicles. Journal of Mechanical Engineering, 2019,55(12):99-120.

[37] Dai Jianguo, Zhao Zhiguo, Liu Taifeng, et al. Research summary of AFPM wheel drive motor. Journal of Hebei University of Science and Technology, 2018,39(1):17-23.

[38] Zheng Danfeng. Summary of Key Technologies of Hub Motor for Pure Electric Vehicle. Automobile and Driving Maintenance (Maintenance Edition), 2018, (1):82.

[39] Xiao Wenwen, Zhang Huanhuan. A summary of ride comfort of electric vehicles driven by in-wheel motors. Journal of Shanghai University of Engineering Science, 2017,31(3):247-251.

[40] Shang Le, Lian Jinyi, Wang Junfeng, et al. Research summary of direct drive technology of hub motor for pure electric vehicles. Automotive Practical Technology, 2018,44(3):7-11.

[41] Xue Yanwei. Research on Four-wheel Independent Steering by Wire Control Strategy of Electric Vehicle. Shaanxi: Xi'an University of Technology, 2017.

[42] Zheng Lu, Wang Zhencheng, Zhao Yongjie, et al. Research on automobile four-wheel steering based on crowd search optimization PID control. Mechanical Design and Manufacturing, 2019, (10):185-188.

[43] Guo cunhan, Miao Xiaodong, Zhang Yuhang, et al. Path following control of four-wheel steering vehicles. Journal of Nantong University (Natural Science Edition), 2018,17(3):23-28.

[44] Zeng Guiling, Ling Jiang, Zhang Yuming, et al. Path following control of four-wheel steering vehicle based on two-point preview. Journal of Guilin Institute of Aerospace Technology, 2019,24(2):204207.

[45] Guo zimeng, Xin Qingqing, Zhang wanzhi, et al. Analysis of handling stability of four-wheel steering vehicle based on Matlab/Simulink. Mechanical engineer, 2018, (8):40-42,45.

[46] Zhang Tingfang, Zhang Chaoxin, He Xinyi, et al. Optimal control of four-wheel steering vehicle based on variable transmission ratio by wire. Mechanical Design and Manufacturing, 2018, (1):133-136.

[47] Nanjing University of Aeronautics and Astronautics. A four-wheel steering vehicle by wire: CN201820809191. X.2019-01-15.

[48] Xu Linna. Research on steering stability control of four-wheel independent drive electric vehicle. Liaoning: Dalian University of Technology, 2019. 
[49] He Haoran, Shan Peng, Li Gang, et al. Research on automobile four-wheel steering control strategy. Automotive Practical Technology, 2016, (1):32-34.

[50] Li Detao. Research on Lateral Stability Control of Four-wheel Independent Steering Drive Electric Vehicle. Jilin: Changchun University of Technology, 2019.

[51] Changchun University of Technology. A stability control method of four-wheel steering vehicle based on time-varying dynamic model: CN201810735047.0.2018-12-21.

[52] Zhang Kai. Four-wheel steering system based on automotive electronic control technology. Electronic Technology and Software Engineering, 2016, (023).

[53] Lei Yongqiang, Li Gang. Research on four-wheel steering control and simulation of four-wheel independent drive electric vehicle. Automotive Practical Technology, 2015, (12):13-14.

[54] Zhou Peng. Study on the handling and stability control strategy of four-wheel steering vehicle [D]. Chongqing University of Technology, 2018.

[55] Liu Zhao. Development and prospect of automobile four-wheel steering system. Automotive Practical Technology, 2015, (4):23-25,39.

[56] Fu Rao. Research on automobile four-wheel steering dynamics and control. Hubei: Wuhan University of Technology, 2007.
[57] Qian Bin. Research on Modeling and Simulation of Automobile Four-wheel Steering. Anhui: Hefei University of Technology, 2009.

[58] Wang Dongming. Research and development of automobile four-wheel steering system. Shanghai Automotive, 2003 (10): 24-27.

[59] Wei Dong. Research on four-wheel steering system with independent control of rear wheels. Chongqing: Chongqing Jiaotong University, 2011.

[60] Wu Huazhi, Wei Xiaojing. Graphic analytical method for steering trapezoidal mechanism of wheeled vehicles. Engineering Machinery, 1999,30 (8): 18.20.

[61] Zhang Liang. Nissan electric steering: stable and smooth running on bumpy roads. Automobile Maintenance, 2014 (3): 6-7.

[62] Wang Jiansheng. Analysis of four-wheel steering motion law of automobile Special Purpose Vehicle, 2004 (5): 23-25.

[63] Guo Konghui, Zha Hao. Development of four-wheel steering control method. China Mechanical Engineering, 1998,9 (5): 73-75.

[64] Bian Lijian, Liu Zhaodu, Qi Zhiquan. Development of automotive active safety electronic technology. China Integrated Circuit, 2004,13 (7): 33.37. 